\title{
Structural diversity in sodium doped water trimers $\dagger$
}

\author{
Richard M. Forck, Johannes M. Dieterich, Christoph C. Pradzynski, Anna L. Huchting, \\ Ricardo A. Mata and Thomas Zeuch*
}

Received 3rd April 2012, Accepted 8th May 2012

DOI: $10.1039 / \mathrm{c} 2 \mathrm{cp} 41066 \mathrm{~d}$

The structures of sodium doped water trimers are characterized on the basis of their infrared action spectra in the $\mathrm{OH}$-stretching region and a global optimization approach to identify the lowest energy minima. The most stable structure is an open ring with two contacts of terminal water molecules to the $\mathrm{Na}$ atom. This structure explains the dominating feature in the IR depletion spectrum around $3410 \mathrm{~cm}^{-1}$. Three additional isomer classes were found in an energy window of $12 \mathrm{~kJ} \mathrm{~mol}^{-1}$ with vertical ionization energies ranging from $\sim 3.83 \mathrm{eV}$ to $\sim 4.36 \mathrm{eV}$. These structures show different hydrogen bonding and sodium coordination patterns and are identified by specific spectral features in the IR spectra. The significant abundance of closed rings with an external $\mathrm{Na}$ atom, resembling the undoped water trimer, suggests that for larger clusters the picture of the sodium atom being situated on the cluster surface seems adequate.

Neutral clusters of water and ammonia being doped by a single sodium atom were considered as microsolutions featuring both the solvation of the sodium atom and the emergence of solvated electrons. $^{1-4}$ The latter was considered to be the main difference to similar clusters with cationic sodium and other alkali metals. ${ }^{1,5}$ Numerous experimental studies on alkali metal doped clusters have been reported in the literature,,$^{2,3,6-10}$ most of them applying photoionization spectroscopy and focussing on the solvents water and ammonia. ${ }^{2,3,7,9-11}$ In this context the unusual IP constancy of $\mathrm{Na}\left(\mathrm{H}_{2} \mathrm{O}\right)_{n}$ clusters levelling off around $3.2 \mathrm{eV}$ for $n \geq 4$, which is different from $\mathrm{Na}\left(\mathrm{NH}_{3}\right)_{n}$ clusters, ${ }^{2,3,7}$ became an issue being discussed controversially until today. ${ }^{2,4,9,10,12,13}$ To solve the controversy the structures of clusters, whose properties dominate the photoionization experiments, have to be revealed. For $\mathrm{Na}\left(\mathrm{NH}_{3}\right)_{n}$ and $\mathrm{Li}\left(\mathrm{NH}_{3}\right)_{n}$ evidence for the alkali metal solution came from electronic and infrared spectroscopy.,14,15 For $\mathrm{Na}\left(\mathrm{H}_{2} \mathrm{O}\right)_{n}$ clusters much less spectroscopic information is available. The depletion spectra generated by electronic excitation indicated the presence of solvated electrons by a sharp drop of excitation energies from $n=1$ to $n=3 .{ }^{6}$ For larger clusters $(n=8,16,20)$ IR spectra were in agreement with scaled harmonic predictions of clusters with solvated $\mathrm{Na}$ atoms. ${ }^{16,17}$ However, it was observed in MD simulations that sodium doping can lead to $\mathrm{Na}$ attachment under pick-up conditions. ${ }^{18}$

Institut für Physikalische Chemie, Universität Göttingen, Tammannstr. 6, 37077 Göttingen, Germany. E-mail: tzeuch1@gwdg.de

$\dagger$ Electronic supplementary information (ESI) available. See DOI: $10.1039 / \mathrm{c} 2 \mathrm{cp} 41066 \mathrm{~d}$

From the theoretical perspective, the results were not unequivocal or even mutually exclusive. In most studies structures where the sodium atom is completely or partly solvated were considered to dominate. ${ }^{4,10,12,19}$ Then again Hashimoto et al. found that structures in which the sodium atom is situated on the surface of water clusters become dominant with increasing $n \cdot{ }^{13,20}$ Hence they postulated the dominance of surface structures with similar vertical ionization potentials (VIPs) to be responsible for the IP constancy. This idea was not followed up in later theoretical work, where density functional theory was used. ${ }^{4,21}$ In these studies, structures with surface $\mathrm{Na}$ atoms were not considered to be competitive, which is different from the $\mathrm{Na}-$ methanol and $\mathrm{Na}$-ethanol systems. ${ }^{9,22}$ In order to explain the constant IP for $n \geq 4$ Gao et al. proposed contributions of autoionization to the photoionization process. $^{4,9}$ The inconclusive literature reports clearly suggest that the composition of the different structure types in the molecular beam needs to be known for better understanding the IP evolution. This point is the focus of the present work.

The clusters were generated in a molecular beam machine with a reflectron time-of-flight mass spectrometer (RETOF) as described elsewhere in detail. ${ }^{23} \mathrm{~A}$ water cluster beam is skimmed and crosses an atmosphere of sodium vapor created in a pick-up cell (cell temperature $490 \mathrm{~K}$ ). The sodium doped clusters are detected by photoionization, mass resolved in the RETOF and the ions are recorded by a multi channel plate. Two typical cluster size distributions are shown in Fig. 1. IR spectra in the $\mathrm{OH}$ stretching region $\left(3000-3800 \mathrm{~cm}^{-1}\right)$ were generated by IR excitation (using a tuneable solid state LaserVision IR laser system) of the clusters and subsequent photoionization at 308 nm (excimer laser, Lambda Physics LPX200i) or $360 \mathrm{~nm}$

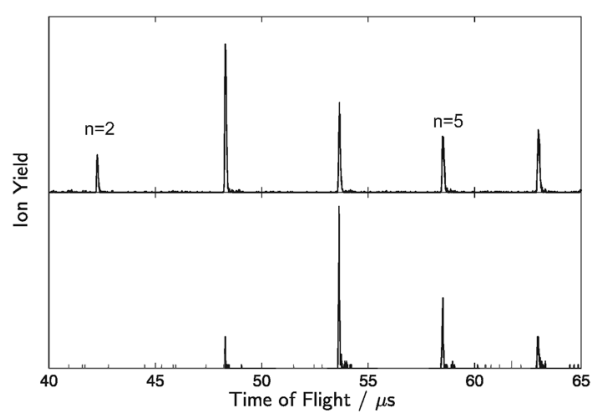

Fig. 1 TOF spectrum at $308 \mathrm{~nm}$ (upper panel, unseeded expansion; $T=320 \mathrm{~K}$ ) and $360 \mathrm{~nm}$ (lower panel, $T=320 \mathrm{~K}$; Ar seeded expansion at 2 bar stagnation pressure). 
(dye laser, Lambda Physics LPD3000). The spectra were recorded with a $10 \mathrm{~cm}^{-1}$ stepwidth and the frequencies were alligned by comparison with FTIR-spectra of polystyrol and polyethylene foils. The IR spectra are smoothed by averaging three neighbouring points $(25 \%, 50 \%, 25 \%)$. This procedure is iterated three times for the lines.

The IR spectrum is generated by exploiting two effects: when the photoionization is performed above or close to the ionization potential (IP) of an isomer, the dominating effect of IR excitation is a signal decrease due to photodestruction of the cluster. When the photoionization is performed below the IP of an isomer, the main effect can also be signal enhancement since heating of the cluster leads to increased abundances of clusters with lower IPs. For $\mathrm{Na}\left(\mathrm{CH}_{3} \mathrm{OH}\right)_{4}$ it was experimentally demonstrated and theoretically rationalized that both effects give similar IR spectra either in signal enhancement or depletion. ${ }^{24}$ In the same work it was shown that fragmentation processes can be controlled and isomer specific depletion and enhancement signals can be separated when expansion conditions and photoionization wavelength are varied such that either effect dominates the IR action spectrum.

In order to support the experimental findings, electronic structure calculations were carried out to characterize the minima of $\mathrm{Na}\left(\mathrm{H}_{2} \mathrm{O}\right)_{3}$ clusters. Given the many conformers such a system can adopt (in a relatively small energy range), starting structures with different bonding motifs were generated. This starting pool of structures was extended through a genetic algorithm (GA) based global optimization. ${ }^{25,26}$ These algorithms have proven to be an efficient tool for minima search in other studies dealing with water clusters. ${ }^{27}$ We made use of the implementation in the OGOLEM framework with a pool-based algorithm and a phenotype crossover operator. ${ }^{28,29}$ The chosen level of theory was B3LYP $/ 6-31+\mathrm{G}^{* *}$ as implemented in the Orca program package. ${ }^{30,31}$ Although this level of theory is relatively inexpensive in the context of electronic-structure based methods, the number of local optimizations necessary for a complete global minimum search is in the regime of multiple thousands. Two independent global optimization runs were carried out. A pool of 100 individuals was optimized for 6000 global optimization steps, yielding a converged solution. A diversity threshold of $1 \times 10^{-4} E_{h}$ was used to avoid premature convergence of the genetic pool. For each structure obtained (within an energy threshold of $50 \mathrm{~kJ} \mathrm{~mol}^{-1}$ from the global minimum), frequencies were computed and scaled by a factor of 0.963 (ZPVEs: 0.9815). In order to confirm the accuracy of the computed cluster energies, higher-level wavefunction calculations were carried out. The restricted open-shell coupled-cluster single and doubles with perturbative triples excitations method [RHF-RCCSD(T)] was used in combination with the aug-cc-pVTZ basis for $\mathrm{O}$ and $\mathrm{H}$ and aug-cc-pCVTZ for the $\mathrm{Na}$ atom. ${ }^{32-34}$ The only electrons removed from the correlation calculation were the 1s electrons of $\mathrm{O}$ and $\mathrm{Na}$. A comparison between the RHF-RCCSD(T) and B3LYP electronic energies is given in the $\mathrm{ESI} \dagger$ and shows relatively good agreement between the two methods. This supports the use of the lower level method in the minima search.

The lowest-lying minima were grouped into different classes according to the $\mathrm{Na}-\mathrm{O}$ contacts $(r(\mathrm{Na}-\mathrm{O}) \leq 3.0 \AA)$ and water hydrogen-bonds (HB). The criteria used for the HB
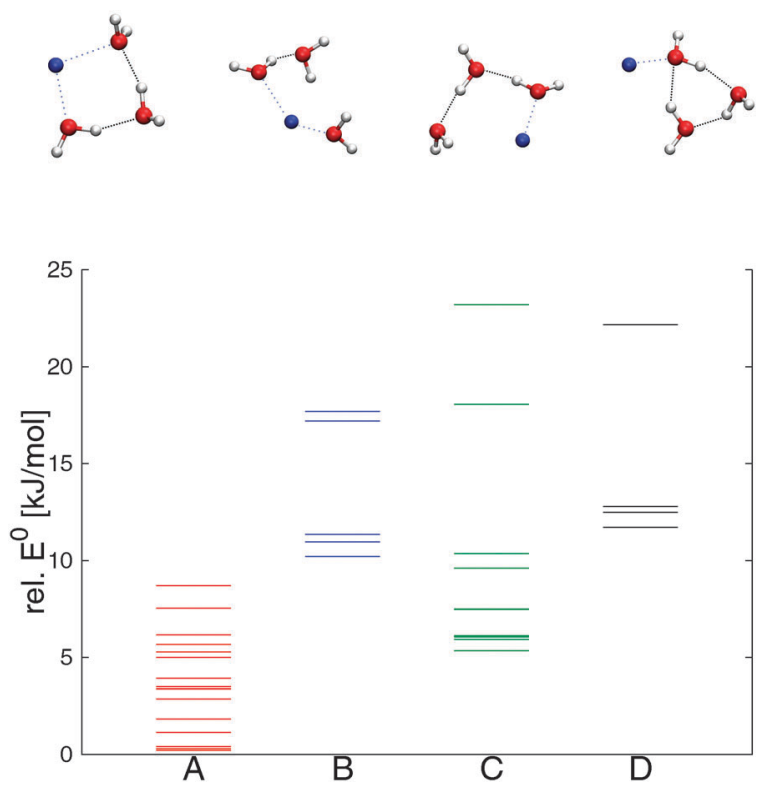

Fig. 2 Energetic ordering of the lowest lying minima for the $\mathrm{Na}\left(\mathrm{H}_{2} \mathrm{O}\right)_{3}$ system, divided according to classes (as described in the text). Structures are shown for the most stable conformer of each class.

were $r(\mathrm{O}-\mathrm{O}) \leq 3.5 \AA, r(\mathrm{O} \cdots \mathrm{H}) \leq 2.5 \AA$ and the angle $\gamma(\mathrm{H}-\mathrm{O} \cdots \mathrm{O}) \leq 45^{\circ}$. Four classes were identified: (A) with $2 \mathrm{Na}-\mathrm{O}$ contacts and $2 \mathrm{HB}$, (B) with $2 \mathrm{Na}-\mathrm{O}$ contacts and $1 \mathrm{HB},(\mathrm{C})$ with $1 \mathrm{Na}-\mathrm{O}$ contact and $2 \mathrm{HB}$ as well as a fourth class (D) with $1 \mathrm{Na}-\mathrm{O}$ contact and $3 \mathrm{HB}$. The pairing, relative energies and the most stable structures for each class are shown in Fig. 2. The energies have been computed at the RHF-RCCSD(T) level of theory, including the scaled DFT ZPVE correction.

The most stable structures are found in class (A), but conformers for every other class are found within $12 \mathrm{~kJ} \mathrm{~mol}^{-1}$ of the lowest minimum. This is a rather small energy gap and justifies the need for extensive minima search. Classes (A) and (C) correspond to open-ring structures, where the water trimer opens to accomodate the $\mathrm{Na}$ atom on one end (forming a fourcenter ring). The difference between the two lies in the orientation of water molecules. In the case of (C) structures, the waters are still oriented as in the water trimer and a terminal water is accomodated such that the interaction with the $\mathrm{Na} 3 \mathrm{~s}$ electron cloud is optimized. This is illustrated in the spin density plots in the ESI. $\dagger$ In (C), only one of the terminal waters has a close $\mathrm{Na}-\mathrm{O}$ contact. In the case of (A), two terminal waters establish a favorable contact with the $\mathrm{Na}$ atom at the expense of a $\mathrm{HB}$ and solvated electron interactions. The (D) class shows a closed water ring with the Na docking to the structure. In the three lowest conformers, the ring structure is close to that of the pure water trimer. The (D) structure which is higher in energy has a double HB acceptor water molecule. The (B) structures show 2 strong $\mathrm{Na}-\mathrm{O}$ contacts, one to a water dimer, the other to a loose water molecule.

In order to complement the IR data, vertical ionization potentials (VIP) were also computed at the RHF-RCCSD(T) level of theory (using the same triple zeta quality basis set). The values are discussed according to the classes used in Fig. 2. Classes (A) and (B) show the smallest VIP, $3.96 \pm 0.05$ and $3.83 \pm 0.05 \mathrm{eV}$ respectively (averaged value with the error bar 
given by the standard deviation). Class (C) has a higher VIP of $4.36 \pm 0.13 \mathrm{eV}$, class (D) $4.19 \pm 0.17 \mathrm{eV}$. The values indicate a correlation between the number of $\mathrm{Na}-\mathrm{O}$ contacts and the VIP values.

Previous theoretical results on the $\mathrm{Na}\left(\mathrm{H}_{2} \mathrm{O}\right)_{3}$ system predicted the IP appearance at about $3.44 \mathrm{eV},{ }^{10} 0.4 \mathrm{eV}$ below our lowest averaged value (class B). This disagreement is due to the absence of structures with $3 \mathrm{Na}-\mathrm{O}$ contacts in our pool. We have performed additional calculations on the trigonal planar isomer (three waters at the vertices aligned away from the $\mathrm{Na}$ in the center) which has been considered in previous studies. ${ }^{13}$ The former is $16.1 \mathrm{~kJ} \mathrm{~mol}^{-1}$ higher in electronic energy, but ZPVE corrections bring the difference down to $5.7 \mathrm{~kJ} \mathrm{~mol}^{-1}$. Since our search was based on electronic energy values, it is easy to understand why such a class has been less represented. Our results, nevertheless, show that it could compete with classes $\mathrm{A}$ and $\mathrm{B}$. This structure has a VIP value of $3.43 \mathrm{eV}$ at the RHF-RCCSD(T) level of theory and explains the discrepancy.

In Fig. 3 IR action spectra for $\mathrm{Na}\left(\mathrm{H}_{2} \mathrm{O}\right)_{3}$ are shown for different experimental conditions in comparison with predicted IR spectra of isomer classes (A-D). In the upper panel of Fig. 3 the IR spectra of comparatively cold clusters (expansion in Ar at $360 \mathrm{~nm}(3.44 \mathrm{eV})$ are shown. Between 3000 and $3800 \mathrm{~cm}^{-1}$ only signal enhancement is observed. These experimental conditions reveal, as discussed above, spectral features of abundant structures with VIPs significantly above the appearance IP. ${ }^{24}$ In the panel below the spectra of class (C) and (D) with the highest VIPs are depicted. The comparison shows that all features

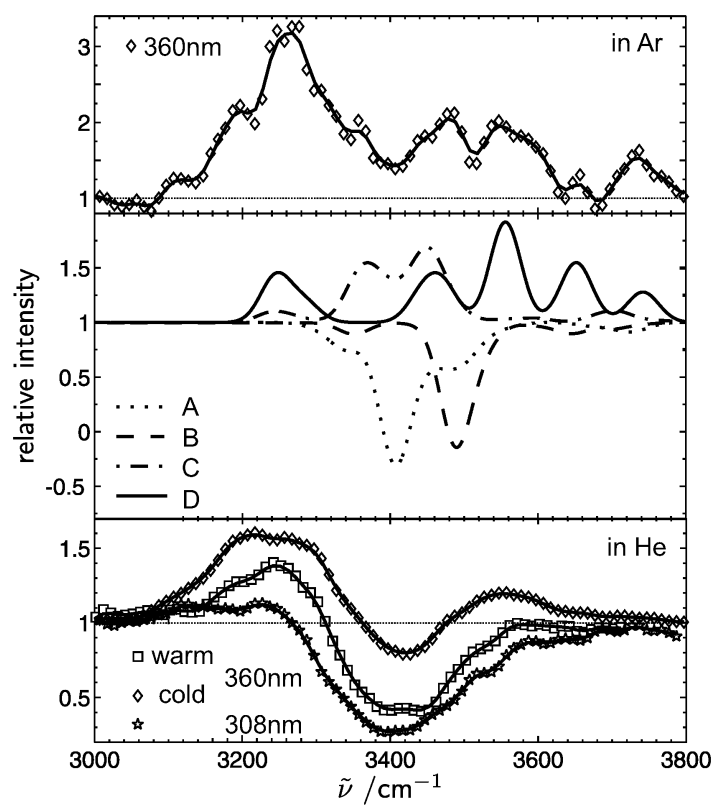

Fig. 3 Infrared spectra of $\mathrm{Na}\left(\mathrm{H}_{2} \mathrm{O}\right)_{3}$ at different expansion conditions and ionizing photon energies. Upper panel: photoionization at $360 \mathrm{~nm}$, Ar seeded expansion at $320 \mathrm{~K}$ and 2 bar stagnation pressure. Lower panel: upper trace: photoionization at $360 \mathrm{~nm}$, He seeded expansion at $320 \mathrm{~K}$ and 2 bar stagnation pressure. Middle trace: photoionization at $360 \mathrm{~nm}$, unseeded expansion at $320 \mathrm{~K}$. Lower trace: photoionization at $308 \mathrm{~nm}$, He seeded expansion at $320 \mathrm{~K}$ and 2 bar stagnation pressure. The middle panel shows predicted IR spectra for isomer classes A-D. The intensities are arbitrarily scaled for an easier comparison with the experiment. of class (D) are present. The high frequency oscillators are the free $\mathrm{OH}$ bands of water molecules with $\mathrm{Na}$ contact at $3660 \mathrm{~cm}^{-1}$ and without at $3740 \mathrm{~cm}^{-1}$. At lower frequency hydrogen bonded oscillators absorb with characteristic red-shifts depending on the position in the ring. The $\mathrm{OH}$ oscillator accepting an $\mathrm{HB}$ and the $\mathrm{Na}$ atom shows a characteristic strong red shift. The water molecule with the highest distance to the sodium corresponds to the feature around $3480 \mathrm{~cm}^{-1}$. The hydrogen bonded oscillator with the highest frequency belongs to the $\mathrm{HB}$ pointing to the $\mathrm{Na}$ accepting water. This finding is similar to $\mathrm{Na}\left(\mathrm{CH}_{3} \mathrm{OH}\right)_{4}$ in cold expansions. ${ }^{24}$ We note that Ar coating and evaporation after $\mathrm{Na}$ pick-up may facilitate the formation of isomer class (D). However, contributions of this effect have not been observed.

We note that class (D) cannot explain the significant absorption between 3300 and $3450 \mathrm{~cm}^{-1}$ because only closed ring structures with a single $\mathrm{Na}$ contact are competive. This is different to the sodium doped methanol tetramer and pentamer, where ring structures with one and two $\mathrm{Na}$ contacts can explain the broad absorption features at low frequencies. ${ }^{24}$ In the case of $\mathrm{Na}\left(\mathrm{H}_{2} \mathrm{O}\right)_{3}$ most likely isomer class $(\mathrm{C})$, which shows the highest VIP, contributes to low frequency absorption. The reproducible feature at $3340 \mathrm{~cm}^{-1}$ is tentatively assigned to this structure. We note that class (C) is only $5 \mathrm{~kJ} \mathrm{~mol}^{-1}$ less stable than the global minimum. The relatively high energy of class (D) and the dominance of its absorption pattern in the IR spectrum indicates that this cluster type is kinetically trapped during sodium attachment in the pick-up process. A similar observation was recently reported for cationic $\mathrm{Li}^{+}\left(\mathrm{H}_{2} \mathrm{O}\right)_{3,4}$ clusters. ${ }^{35}$

In the lower panel of Fig. 3 IR action spectra for $\mathrm{Na}\left(\mathrm{H}_{2} \mathrm{O}\right)_{3}$ are shown for warmer He expansions at $360 \mathrm{~nm}(3.44 \mathrm{eV})$ and $308 \mathrm{~nm}(4.02 \mathrm{eV})$. These spectra differ significantly at higher frequencies. The strongest depletion feature at $3410 \mathrm{~cm}^{-1}$ corresponds to the strongest feature of the most stable isomer class (A). Increasing the cluster temperature in an unseeded expansion (at $360 \mathrm{~nm}$ ) enhances signal depletion and diminishes signal enhancement upon IR irradiation. Metastable fragment peaks and other features indicating the onset of fragmentation are not observed for $n=3 .^{24}$ The relatively high absorption around $3500 \mathrm{~cm}^{-1}$ indicates the presence of isomer class (B). We note that the effect of including the trigonal planar structure, which explains the appearance IP of $\mathrm{Na}\left(\mathrm{H}_{2} \mathrm{O}\right)_{3}$, on the predicted IR spectrum is negligible. The dominating feature around $3410 \mathrm{~cm}^{-1}$ in the depletion spectrum in He expansions clearly indicates that class (A), which includes the global minimum, dominates among clusters, where the original three ring has opened. At the same time we have clear evidence that kinetic trapping of cyclic water trimers with an external $\mathrm{Na}$ atom is possible even in warm expansions as indicated by the signal enhancement below $3350 \mathrm{~cm}^{-1}$ in He expansions at $360 \mathrm{~nm}$ (see lower panel of Fig. 3).

This study has a number of conclusions: there is now ample evidence that already for $\mathrm{Na}\left(\mathrm{H}_{2} \mathrm{O}\right)_{3}$ a broad distribution of isomers with very different bonding motifs is generated in the $\mathrm{Na}$ pick-up process. The global optimization approach being employed here was able to identify the energetically favorable configurations including the most stable cluster class (A), which was not considered in previous work. The kinetic trapping of higher energy isomers clearly supports the Hashimoto-Morokuma 
hypotheses that structures with the $\mathrm{Na}$ atom situated on the cluster surface contribute to the unusual IP constancy. To settle this issue, larger clusters must be characterized in detail, which is beyond the scope of this work considering the isomer diversity revealed for $n=3$. The high level VIP calculations clearly show that the breadth of the photionization spectra is related to a variety of competitive bonding motifs of sodium doped water clusters. Finally, it has been shown that structures explaining the appearance IP are not necessarily representative for the isomer composition in the molecular beam.

\section{Acknowledgements}

TZ thanks Prof. Martin Suhm and Prof. Udo Buck for fruitful discussions. Funding by the DFG (GRK 782, grant ZE 890-1-1) is gratefully acknowledged.

\section{References}

1 C. P. Schulz, R. Haugstätter, H.-U. Tittes and I. V. Hertel, Phys. Rev. Lett., 1986, 57, 1703-1706.

2 I. V. Hertel, C. Hüglin, C. Nitsch and C. P. Schulz, Phys. Rev. Lett., 1991, 67, 1767-1770.

3 R. Takasu, F. Misaizu, K. Hashimoto and K. Fuke, Surf. Rev. Lett., 1996, 3, 353-357.

4 B. Gao and Z. Liu, J. Chem. Phys., 2007, 126, 084501.

5 C. J. Weinheimer and J. M. Lisy, Int. J. Mass Spectrom. Ion Processes, 1996, 159, 197-208.

6 C. P. Schulz, C. Bobbert, T. Shimosato, K. Daigoku, N. Miura and K. Hashimoto, J. Chem. Phys., 2003, 119, 11620-11629.

7 C. Steinbach and U. Buck, J. Chem. Phys., 2005, 122, 134301.

8 T. E. Salter, V. A. Mikhailov, C. J. Evans and A. M. Ellis, J. Chem. Phys., 2006, 125, 0343302.

9 I. Dauster, M. A. Suhm, U. Buck and T. Zeuch, Phys. Chem. Chem. Phys., 2008, 10, 83-95.

10 R. M. Forck, I. Dauster, Y. Schieweck, T. Zeuch, U. Buck, M. Oncak and P. Slavicek, J. Chem. Phys., 2010, 132, 221102.

11 T. E. Salter and A. M. Ellis, J. Phys. Chem. A, 2007, 122, 4922-4926.
12 R. N. Barnett and U. Landmann, Phys. Rev. Lett., 1993, 70, $1775-1778$.

13 K. Hashimoto and K. Morokuma, J. Am. Chem. Soc., 1994, 116 , 11436-11443.

14 T. E. Salter, V. A. Mikhailov and A. M. Ellis, J. Chem. Phys., 2006, 125, 0343302.

15 L. Varriale, N. M. Tonge, N. Bhalle and A. M. Ellis, J. Chem. Phys., 2010, 132, 161101.

16 C. Steinbach and U. Buck, J. Phys. Chem. A, 2006, 110, $3128-3131$.

17 U. Buck, I. Dauster, B. Gao and Z. Liu, J. Phys. Chem. A, 2007, 111, 12355-12362.

18 L. Cwiklik, U. Buck, W. Kulig, P. Kubisiak and P. Jungwirth, J. Chem. Phys., 2008, 128, 154306.

19 T. Tsurusawa and S. Iwata, J. Phys. Chem. A, 1999, 103, 6134-6141.

20 K. Hashimoto, S. He and K. Morokuma, Chem. Phys. Lett., 1993, 206, 297-304.

21 Y. Ferro and A. Allouche, J. Chem. Phys., 2003, 118, 10461-10469.

22 R. Forck, I. Dauster, U. Buck and T. Zeuch, J. Phys. Chem. A, 2011, 115, 6068-6076.

23 C. Schütte and U. Buck, Int. J. Mass Spectrom., 2002, 220, $183-192$.

24 R. M. Forck, C. C. Pradzynski, S. Wolff, M. Oncak, P. Slavicek and T. Zeuch, Phys. Chem. Chem. Phys., 2012, 14, 3004-3016.

25 B. Hartke, Wiley Interdiscip. Rev.: Comput. Mol. Sci., 2011, 1, 879-887.

26 R. L. Johnston, Dalton Trans., 2003, 4193-4207.

27 B. Bandow and B. Hartke, J. Phys. Chem. A, 2006, 110, $5809-5822$.

28 J. M. Dieterich and B. Hartke, Mol. Phys., 2010, 108, 279-291.

29 J. M. Dieterich and B. Hartke, J. Comput. Chem., 2011, 32, $1377-1385$.

30 A. D. Becke, J. Chem. Phys., 1993, 98, 5648-5652.

31 F. Neese, Orca: An ab initio, DFT and semiempirical electronic structure package, version 2.8, 2011.

32 H.-J. Werner, P. J. Knowles, G. Knizia, F. R. Manby and M. Schütz et al., MOLPRO, version 2010.1, a package of ab initio programs, 2010, see http://www.molpro.net.

33 T. Dunning, J. Chem. Phys., 1989, 90, 1007-1024.

34 D. Woon and T. Dunning, J. Chem. Phys., 1995, 103, 4572-4586.

35 O. Rodriguez and J. M. Lisy, J. Phys. Chem. Lett., 2011, 2, $1444-1448$. 